\title{
BLUE-INFRARED NARROW-BAND PHOTOMETRY FROM OBJECTIVE PRISM SPECTRA
}

\author{
A. CASSATELLA, P. MAFFEI, and R. VIOTTI
}

Laboratorio di Astrofisica Spaziale - Frascati, Italy

(Read by L. Gratton)

\begin{abstract}
A technique for narrow-band photometry in the $3600-8400 \AA$ spectral region from objective prism spectra is briefly described and applied to the Hyades.
\end{abstract}

For the purpose of making a quantitative study of a large number of low dispersion stellar spectra in young associations, a technique for the rapid reduction of spectra using the new digitized microphotometer MI.DI. and the IBM 1130 computer of the Laboratorio di Astrofisica Spaziale has been developed and applied, as a preliminary test, to the Hyades. A detailed description of the microphotometer and of its applications is given by Gratton et al. (1971).

The work is based on 8 IN Kodak plates obtained with the 65/90/210-cm Schmidt telescope of the Asiago Astrophysical Observatory using a $4^{\circ}$ objective prism which gives a reciprocal dispersion of $608 \AA \mathrm{mm}^{-1}$ at $\mathrm{H} \gamma$. Half of the plates were hypersensitized with a flash lamp in order to reach the faintest stars - the average gain in the limiting magnitude being about $1^{m} .5$ or better which makes the use of hypersensitized plates rather convenient, provided the number of observations be doubled in order to have the same $S / N$ ratio of the normal plates.

The photometric calibration was made with the Asiago spectrosensitometer on IN plates taken from the same box of plates used for the observations. The observational and calibration material was processed all together. Each calibration was read by the microphotometer at several different wavelengths and all the recordings were elaborated using a suitable program which gives the single characteristic curves and the averaged curve together with the internal accuracy of the calibration itself. It was found that the characteristic curve is not too sensitive to the wavelength and to the exposure time (in the range $10 \mathrm{~min}$ to $2 \mathrm{~h}$ ), and does not vary between the plates of the same box; but it is very different between normal and hypersensitized plates (Cassatella and Viotti, 1971).

The correction for the selective instrumental sensitivity and the atmospheric extinction was made in the usual way by recording a number of standard Hyades stars among those whose monochromatic fluxes were measured with a photoelectric scanner by Oke and Conti (1965). The plate corrections $c(\lambda)$ were derived by comparing the observed photographic monochromatic magnitudes with the photoelectric ones. The procedure is described in detail in Figure 1 which shows the block diagram of the reduction program. Figures 2, 3 and 4 give the uncorrected monochromatic magnitudes for three stars of the Hyades. 


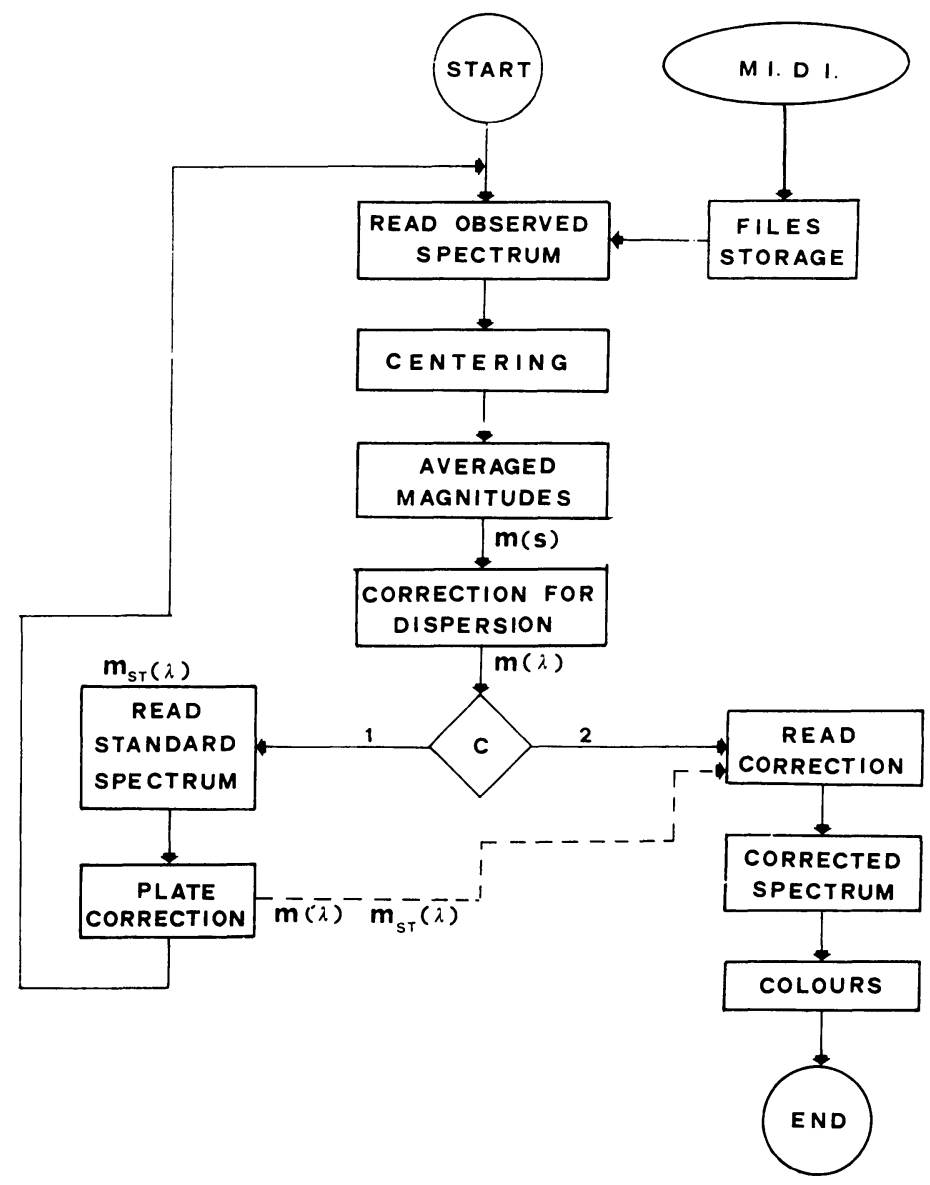

Fig. 1. Block diagram of the reduction programs.

Since more than two standard stars are present in each plate, a photometric calibration of the plate could be derived directly by comparing the plate densities at a given wavelength with the corresponding monochromatic magnitudes of the standard stars. It was found that the slope of the characteristic curves in this manner are practically the same as those computed from the calibration plates. Since in the latter case we have a much larger number of points for drawing the characteristic curves, it was found more convenient to follow in this study the latter method.

The following Figure 5 gives the corrections $c(\lambda)$ derived for one IN plate. As soon as the $c(\lambda)$ of each plate were computed, the second part of the program in Figure 1 was used for the reduction of the spectra, and the corrected magnitudes were derived. Since one of the applications of this program is the determination of the infrared excess for stars in young associations, a blue-infrared color index $C \gamma$ was introduced defined as

$$
c_{\gamma}=m_{4464}-\frac{1}{2}\left(m_{7100}+m_{8400}\right),
$$




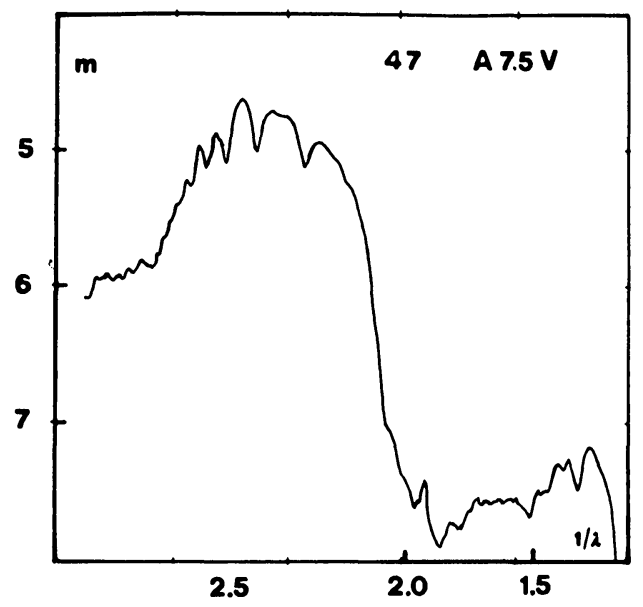

Fig. 2. Tracing of star $\mathrm{N} 47$ of the Hyades (A7.5V). In ordinates monochromatic magnitudes corrected for the dispersion.

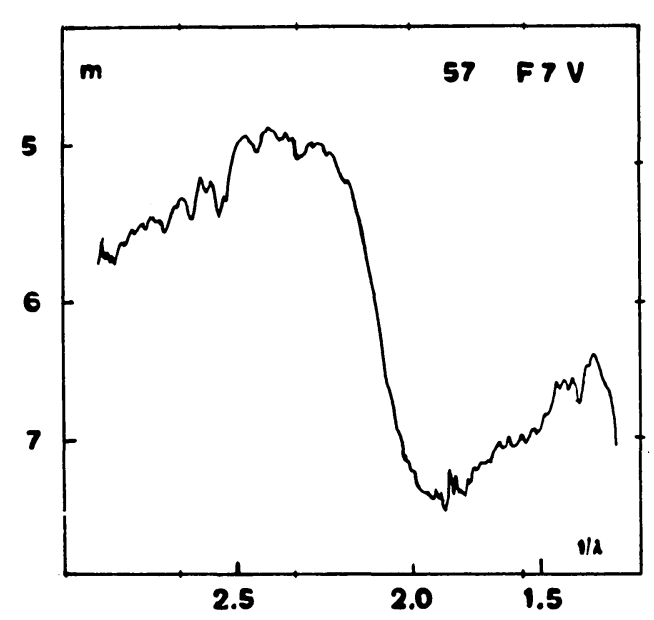

Fig. 3. Tracing of star N 57 (F7V).

where $m_{\lambda}$ are the mean magnitudes in a band centered on $\lambda$. The widths of the blue and of the two infrared bands are $50 \AA$ and $100 \AA$ respectively. The three bands were selected among those measured by Oke and Conti, in such a way to have a maximum plate density and to avoid as much as possible the stellar strong absorption bands and lines and the atmospheric bands.

74 color indices were computed for 30 Hyades stars, and the average $C_{\gamma}$ are plotted in Figures 6 and 7. The mean plate correction for the $C_{\gamma}$ colors was $-2^{m} .33$ and the variation from plate to plate (hypersensitized or not) was no greater than $0^{m} \cdot 2$. The circles are main sequence A6-K8 stars; the triangle is a K0III star ( $\gamma$ Tau). Figure 6 


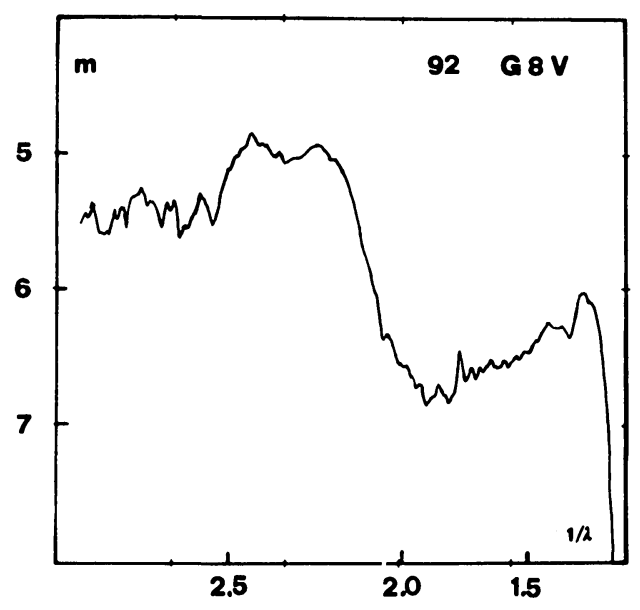

Fig. 4. Tracing of star N $92(\mathrm{G} 8 \mathrm{~V})$.

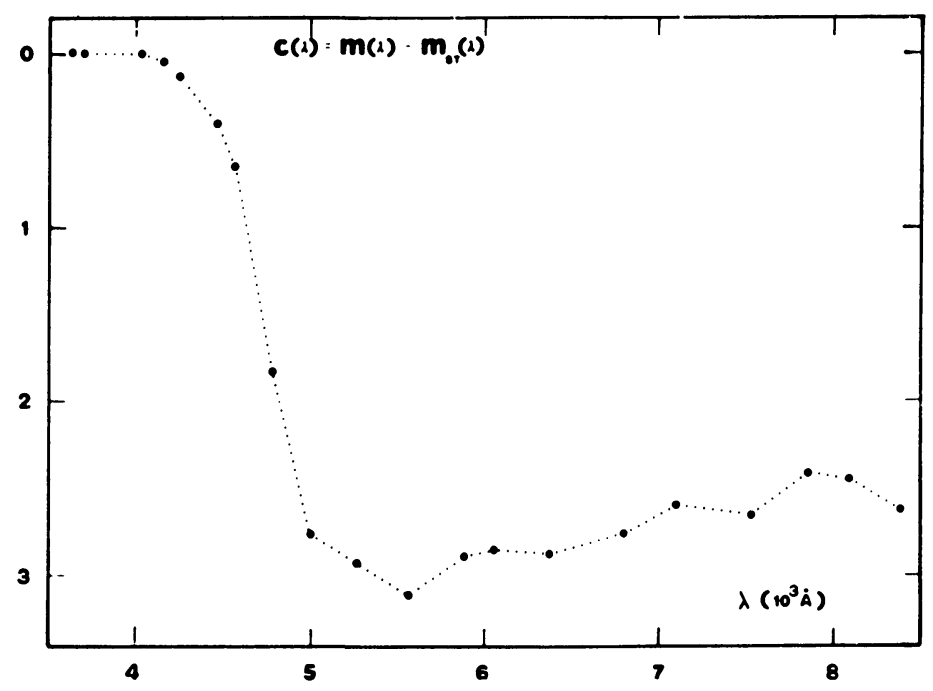

Fig. 5. Plate corrections (in magnitudes) for a single IN plate.

gives the two color diagram $C_{\gamma}, B-V$ for the Hyades; the source for the $B-V$ is Johnson and Knuckels (1955). Figure 7 shows the plot of $C_{\gamma}$ as a function of the spectral type given by Morgan and Hiltner (1965) and by Ramberg (1941). The full lines are the polynomial best fits of the data. The mean scatter of the points from the lines is $0^{m} .05$ and $0^{m} .08$ respectively. The mean error for a single measurement for normal plates is between $0^{m} .04$ and $0^{m} .08$ depending on the faintness of the star, and is about $30-50 \%$ larger for the hypersensitized plates. These errors are in a good agreement with the expected accuracy for photographic photometry with objective prism spectra (see 


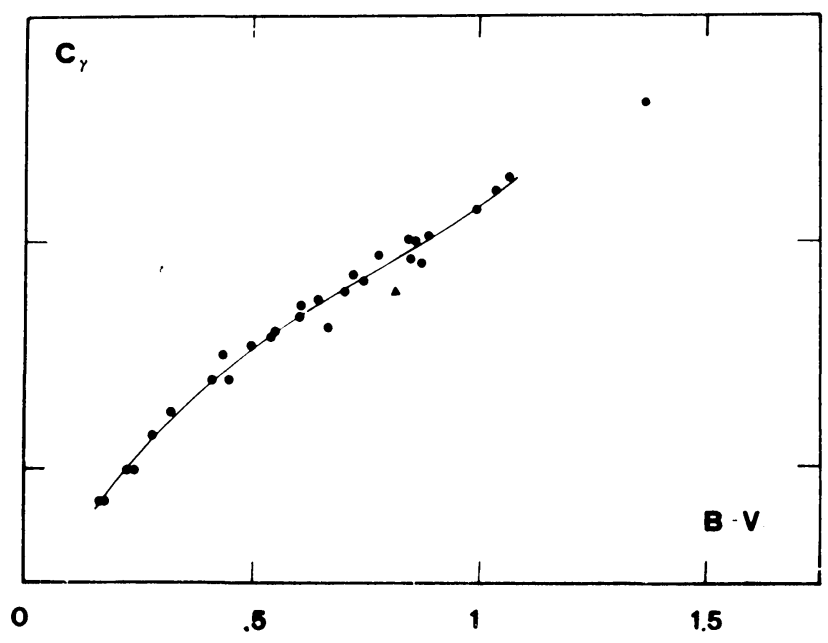

Fig. 6. Two color diagram for the Hyades. (See text for the symbols.)

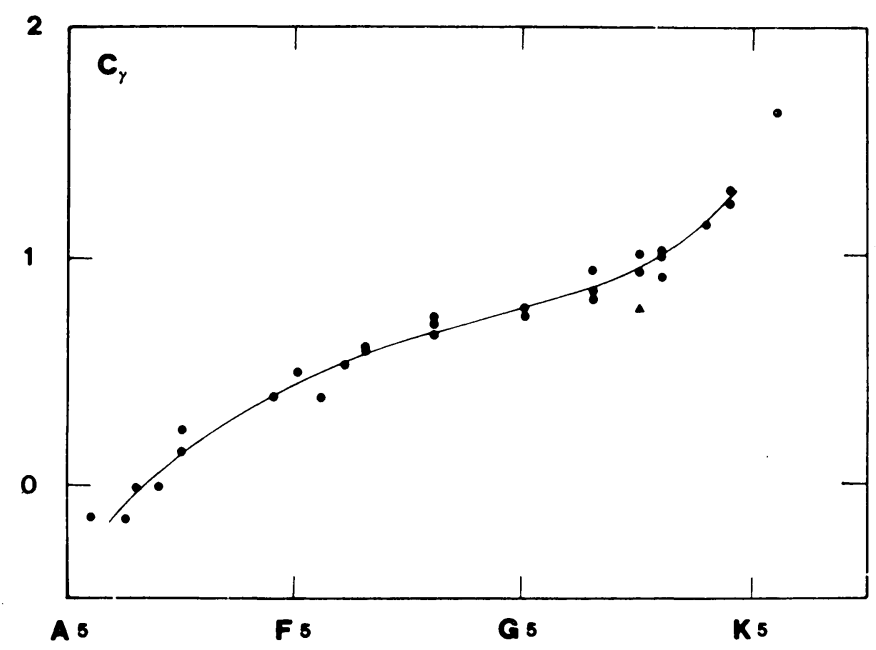

Fig. 7. $C \gamma$-spectral type diagram.

e.g. Gratton, 1939). The larger dispersion in Figure 3 may be accounted for errors in spectral classification. The color magnitude diagrams $V, C_{\gamma}$ and $m(8400), C_{\gamma}$ are given in Figures 8 and 9.

The results in Figures 6-9 show that a satisfactory narrow band photometry can be made with objective prism plates, with the great advantage that one can make simultaneously the photometry of a very large number of stars in several different spectral regions and, in addition, a spectral classification can be provided from a visual inspection of the spectra or, automatically, with a suitable reduction program. For this 


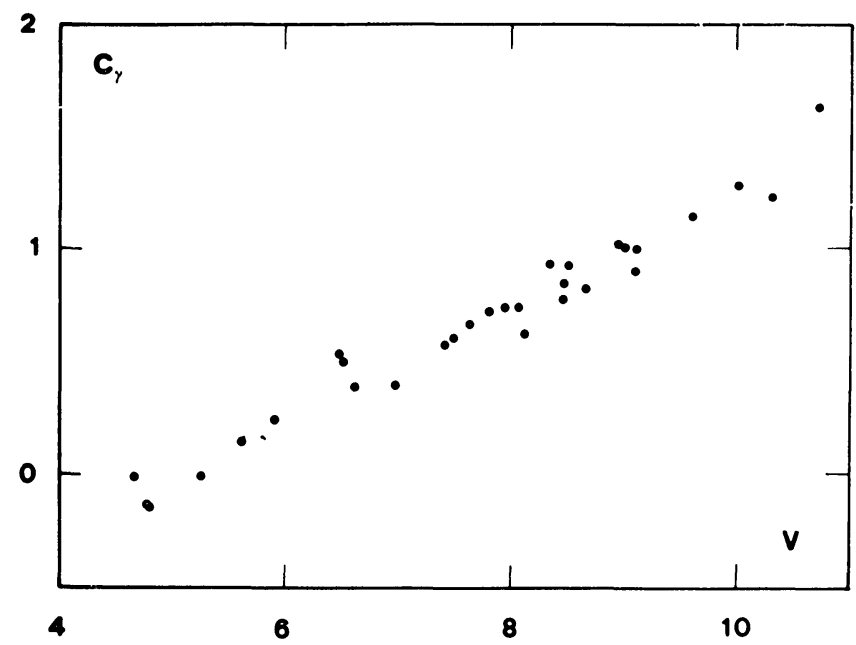

Fig. 8. Color-magnitude diagram.

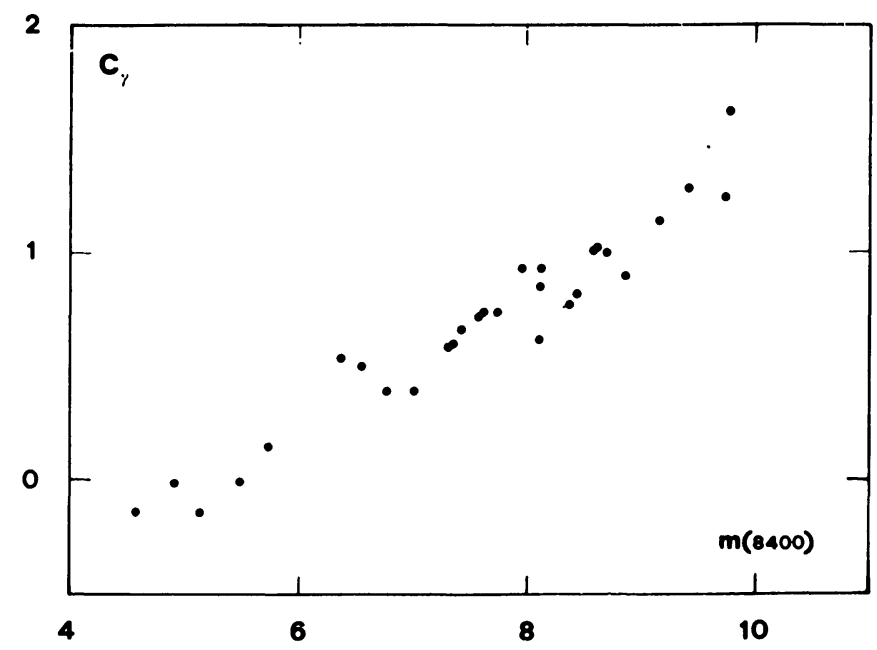

Fig. 9. Color-infrared magnitude diagram.

reason the use of automatic measuring devices allows for a rapid reduction of the observational material. As for example, 100 spectra may be recorded with a digitized microphotometer in a few hours, including all the dead times. The time for the reduction of a single spectrum is 2 min for reading the punched tape, and $1 \mathrm{~min}$ for all the computations. The total time would be drastically reduced by using on-line connection between microphotometer and computer, so that the complete study of an association at the objective prism could be made in less than one day. 


\section{References}

Cassatella, A. and Viotti, R.: 1971, Rapporto Interno No. 23, Laboratorio di Astrofisica Spaziale, Frascati.

Gratton, L.: 1939, Stockholm Obs. Ann. 13, No. 3.

Gratton, L., Martini, A., Martino, E., Natali, G., and Viotti, R.: 1971, Publ. Roy. Obs. Edinburgh 8, 142.

Johnson, H. L. and Knuckéls, C. F.: 1955, Astrophys. J. 122, 209.

Morgan, W. W. and Hiltner, W. A.: 1966, Astrophys. J. 141, 177.

Oke, J. B. and Conti, P. S.: 1966, Astrophys. J. 143, 134.

Ramberg, J. M.: 1941, Stockholm Obs. Ann. 13, No. 9. 